TITLE:

\title{
Primordial nucleosynthesis and hadronic decay of a massive particle with a relatively short lifetime
}

AUTHOR(S):

Kohri, K

CITATION:

Kohri, K. Primordial nucleosynthesis and hadronic decay of a massive particle with a relatively short lifetime. PHYSICAL REVIEW D 2001, 64(4): 043515.

ISSUE DATE:

2001-08-15

URL:

http://hdl.handle.net/2433/50452

RIGHT:

Copyright 2001 American Physical Society 


\title{
Primordial nucleosynthesis and hadronic decay of a massive particle with a relatively short lifetime
}

\author{
Kazunori Kohri \\ Yukawa Institute for Theoretical Physics, Kyoto University, Kyoto, 606-8502 Japan
}

(Received 31 March 2001; published 30 July 2001)

\begin{abstract}
In this paper we consider the effects on big bang nucleosynthesis (BBN) of the hadronic decay of a long-lived massive particle. If high-energy hadrons are emitted near the BBN epoch $\left(t \sim 10^{-2}-10^{2} \mathrm{sec}\right)$, they extraordinarily interconvert the background nucleons with each other even after the freeze-out time of the neutron to proton ratio. Then, the produced light element abundances are changed, and that may result in a significant discrepancy between standard BBN and observations. Especially on the theoretical side, we can now obtain a lot of experimental data on hadrons and simulate the hadronic decay process, executing the numerical code of the hadron fragmentation even in the high energy region where we have no experimental data. Using the computed light element abundances in the hadron-injection scenario, we derive a constraint on the properties of such a particle by comparing our theoretical results with observations.
\end{abstract}

DOI: 10.1103/PhysRevD.64.043515

PACS number(s): 98.80.Cq, 26.35.+c, 98.80.Ft

\section{INTRODUCTION}

Big bang nucleosynthesis (BBN) is one of the most important tools to probe the early universe because it is sensitive to the condition of the universe from $10^{-2} \mathrm{sec}$ to $10^{4} \mathrm{sec}$. Therefore, from theoretical predictions we can indirectly check the history of the universe in such an early epoch and impose constraints on hypothetical particles by observational light element abundances.

Now we have a lot of models of modern particle physics beyond the standard model, e.g., supergravity or superstring theory, which predict unstable massive particles with masses of $\mathcal{O}(100) \mathrm{GeV}-\mathcal{O}(10) \mathrm{TeV}$, such as the gravitino, Polonyi field, and moduli. They have long lifetimes because their interactions are suppressed by inverse powers of the gravitational scale $\left(\propto 1 / M_{\mathrm{pl}}\right)$. These exotic particles may necessarily decay at about the BBN epoch $[T \leqslant \mathcal{O}(1) \mathrm{MeV}]$ if they have already existed in earlier stages. If the massive particles radiatively decay, the emitted high energy photons induce the electromagnetic cascade process. If the decay occurs after BBN starts $t \gtrsim 10^{4} \mathrm{sec}$, the light elements would be destroyed by the cascade photons and their abundances would be changed significantly. Comparing the theoretical prediction of light element abundances with observations, we can impose constrains on the energy density, the mass, and the lifetime of the parent massive particle [1-3]. This subject was also studied in more detail in a recent paper [4].

On the other hand, if the massive particles decay into quarks or gluons near the BBN epoch $10^{-2} \leq t \leq 10^{2} \mathrm{sec}$, it is expected that other important effects are induced. If the high energy quarks or gluons are emitted, they quickly fragment into numerous hadrons. Then, such high energy hadrons are injected into the electromagnetic thermal bath which is constituted of photons, electrons, and nucleons (protons and nucleons) at that time. At first, the high energy hadrons scatter off the background photons and electrons because they are more abundant than the background nucleons. Then, al- most all the kinetic energy of the hadrons is transferred into the thermal bath through the electromagnetic interaction. As a result, it is completely stopped and reaches kinetic equilibrium. After that time, they scatter off the background $p$ or $n$ through the strong interaction, and they interconvert the background $p$ and $n$ with each other even after the usual freeze-out time of the neutron to proton ratio $n / p$ of the weak interaction. The effect extraordinarily tends to increase $n / p$. Therefore, the produced ${ }^{4} \mathrm{He}$ would be increased in the hadron injection scenario compared to standard big-bang nucleosynthesis (SBBN).

The pioneering investigation of this subject was done by Reno and Seckel [5], and their treatments have been applied to the other subjects $[6,7]$. After their work was published, the experiments of high energy physics became widely developed. Now we can obtain a lot of experimental information about the hadron fragmentation in the high energy region and also simulate the process even in the higher energies where we have no experimental data by executing the numerical code of the hadron fragmentation, e.g., JETSET 7.4 Monte Carlo event generator [8]. In addition, we have more experimental data of the hadron-nucleon cross sections. Concerning BBN computations, it has recently become necessary that we perform a Monte Carlo simulation which includes the experimental errors of the reactions, and then we estimate the confidence levels (C.L.) by performing the maximum likelihood analysis and the $\chi^{2}$ fitting including both the theoretical and the observational errors. Performing the above procedures, we can compare each model in the various parameter sets. With these new developments in the theory, we set bounds to the hadronic decay of long-lived particles. ${ }^{1}$

This paper is organized as follows. In Sec. II we briefly review the current status of the observations and SBBN. In Sec. III we introduce the formulations and computations in

\footnotetext{
${ }^{1}$ For relatively longer lifetimes, there exists an another interesting process that the emitted high energy nucleons destroy the light elements which have already been produced [9].
} 
the hadron injection scenario. In Sec. IV we compare thetheoretical predictions with the observations. Section V is devoted to the summary and conclusions.

\section{CURRENT STATUS OF OBSERVATIONAL LIGHT ELEMENT ABUNDANCES AND SBBN}

\section{A. Current status of observations}

In this section, we briefly summarize the current status of the observational light element abundances. The primordial $\mathrm{D} / \mathrm{H}$ is measured in the high redshift quasistellar object (QSO) absorption systems. Recently new deuterium data was obtained from observation of QSO HS $0105+1619$ at $\mathrm{z}$ $=2.536[10]$. The reported value of the deuterium abundance was relatively low, $(\mathrm{D} / \mathrm{H})^{o b s}=(2.54 \pm 0.23) \times 10^{-5}$. Combined with the previous "low D" data [11], the authors reported that the primordial abundance is

$$
\text { low D: } \quad(\mathrm{D} / \mathrm{H})^{o b s}=(3.0 \pm 0.4) \times 10^{-5} \text {. }
$$

We call this value "low D." On the other hand, Webb et al. obtained a high deuterium abundance in relatively low redshift absorption systems at $\mathrm{z}=0.701$ towards QSO PG1718 +4807 [12],

$$
\text { high D: } \quad(\mathrm{D} / \mathrm{H})^{o b s}=(2.0 \pm 0.5) \times 10^{-4} \text {. }
$$

In these days, Kirkman et al. [13] also observed the clouds independently and obtained new spectra using HST. They claimed that the absorption was not deuterium although there were still some uncertainties. Here we think that it is premature to decide which component is correctly primordial; the possibility of "high D" has not been excluded yet. Therefore, we also consider the possibility of "high D" and include it in our analysis.

The primordial value of ${ }^{4} \mathrm{He}$ is inferred from the recombination lines from the low metallicity extragalactic HII regions. The primordial value of ${ }^{4} \mathrm{He}$ mass fraction $Y$ is obtained to regress to the zero metallicity $\mathrm{O} / \mathrm{H} \rightarrow 0$ for the observational data because it is produced with oxygen in the stars. In these days Fields and Olive reanalyzed the data including the HeI absorption effect [14]. Then they obtained the observational $Y$,

$$
Y^{o b s}=0.238 \pm(0.002)_{s t a t} \pm(0.005)_{s y s t},
$$

where the first error is the statistical uncertainty and the second error is the systematic one. We adopt this value as the observational value of $Y$.

It is widely believed that the primordial abundance of ${ }^{7} \mathrm{Li} / \mathrm{H}$ is observed in the Pop II old halo stars whose temperature is high $T_{\text {eff }} \gtrsim 6000 \mathrm{~K}$ and metallicity is low $[\mathrm{Fe} / \mathrm{H}]$ $\lesssim-1.5$. They have the "plateau" structure of ${ }^{7} \mathrm{Li} / \mathrm{H}$ as a function of the metallicity. We adopt the recent measurements by Bonifacio and Molaro [15]

$$
\begin{aligned}
\log _{10}\left[\left({ }^{7} \mathrm{Li} / \mathrm{H}\right)^{\text {obs }}\right]= & -9.76 \pm(0.012)_{\text {stat }} \pm(0.05)_{\text {syst }} \\
& \pm(0.3)_{\text {add }} .
\end{aligned}
$$

Here we have added the additional uncertainty for fear that the ${ }^{7} \mathrm{Li}$ in halo stars might have been supplemented (by production in cosmic-ray interactions) or depleted (in stars) $[16]^{2}$

\section{B. Current status of SBBN}

Here we show the current status of standard big-bang nucleosynthesis $(\mathrm{SBBN})$. Within recent years, there was a great progress in the experiments of the low energy cross sections for 86 charged-particle reactions by the NACRE Collaboration [19]. In the compilation, 22 reactions are relevant to the primordial nucleosynthesis, and the old data were revised. In particular, of the 22 reactions, seven of them are important for the most elementary process up to mass-7 elements. On the other hand, Cyburt, Fields, and Olive recently reanalyzed the NACRE data and properly derived the $1 \sigma$ uncertainty as a statistics meaning and the renormalization of the center value for each reaction [20]. In addition, they also reanalyzed the four remaining reactions, using the existing data [21-23] and the theoretical prediction (for one reaction) [24]. Their efforts are quite useful for the study of the Monte Carlo simulation in BBN, and it was shown that their treatment is consistent with the other earlier studies adopting the results of NACRE $[25,26]$.

Carrying the Monte Carlo simulation into execution, we adopt the theoretical errors and the center values for 11 elementary nuclear reactions in Ref. [20]. For the error and the center value of a neutron lifetime, we adopt the compilation of the Particle Data Group [27], see Eq. (10). To systematically take account of the uncertainties, we perform the maximum likelihood analysis [3] including both the observational and theoretical errors which are obtained in Monte Carlo simulation. Here we assume that the theoretical predictions of $(\mathrm{D} / \mathrm{H})^{t h}, Y^{t h}, \log _{10}\left[\left({ }^{7} \mathrm{Li} / \mathrm{H}\right)^{t h}\right]$ obey the Gaussian probability distribution functions (p.d.f.'s) with the widths given by the $1 \sigma$ errors. Concerning the observational values, $(\mathrm{D} / \mathrm{H})^{o b s}, Y^{o b s}$, and $\log _{10}\left[\left({ }^{7} \mathrm{Li} / \mathrm{H}\right)^{o b s}\right]$ are also assumed to obey the Gaussian p.d.f.'s.

In Fig. 1 we plot $\chi^{2}$ as a function of baryon to photon ratio, $\eta=n_{B} / n_{\gamma}$, where $n_{B}$ is the baryon number, and $n_{\gamma}$ is the photon number. The solid line (dashed line) represents the case of low D (high D). From this figure, we find that SBBN agrees with the observation of ${ }^{4} \mathrm{He}, \mathrm{D}$, and ${ }^{7} \mathrm{Li}$ very well at more than $95 \%$ C.L., and we obtain $\eta=5.6_{-0.8}^{+0.9}$ $\times 10^{-10}\left(\eta=1.8_{-0.5}^{+1.6} \times 10^{-10}\right)$ for low $\mathrm{D}$ (high D) at $95 \%$ C.L. Using the relation $\Omega_{B} h^{2}=3.63 \times 10^{7} \eta\left(T_{0} / 2.725 K\right)$, we obtain

\footnotetext{
${ }^{2}$ These days, however, it was claimed that there is a significant Li-Fe trend in the low metallicity region [17]. In addition, Ryan et al. [18] assumed that this trend is due to the cosmic ray interactions, and they inferred the primordial value is ${ }^{7} \mathrm{Li} / \mathrm{H}=\left(1.23_{-0.32}^{+0.68}\right)$ $\times 10^{-10}$. Because we cannot make a judgment on the above discussions, for the moment we adopt the value in Eq. (4) with large uncertainties in this paper.
} 


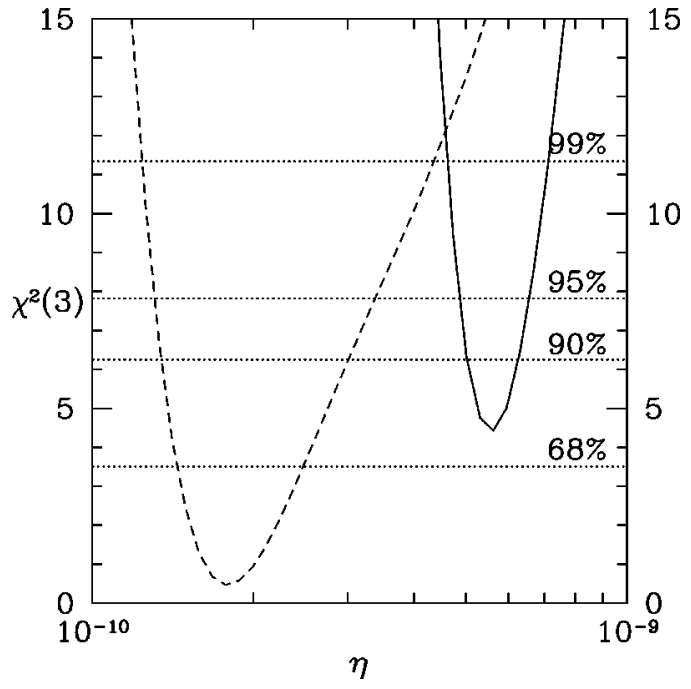

FIG. 1. Plot of $\chi^{2}$ as a function of baryon to photon ratio ( $\eta$ $=n_{B}\left(n_{\gamma}\right)$. The solid line (dashed line) represents the case of low $\mathrm{D}$ (high D).

$$
\Omega_{B} h^{2}= \begin{cases}0.0203_{-0.0029}^{+0.0033} & (\text { for low D) } \\ 0.0065_{-0.0018}^{+0.0058} & \text { (for high D) },\end{cases}
$$

at $95 \%$ C.L., where $\Omega_{B}$ is the baryon density parameter, $h$ is the normalized Hubble parameter as $H_{0}=100 \mathrm{~h} \mathrm{~km} /$ sec/Mpc, and $T_{0}$ is the present temperature [27]. Under these circumstances, we can check the nonstandard scenario comparing the predictions of the BBN computations with observations.

\section{HADRONIC DECAY AND BBN}

In this section, we discuss the hadron-injection effects on the history of the universe near BBN epoch $(t$ $\left.=10^{-2}-10^{4} \mathrm{sec}\right)$. Here we consider the case that the unstable massive particle " $X$ " has some decay modes into quarks and gluons, and as a result it induces the late-time hadron injection.

\section{A. Time scale of the interactions}

If the quarks and gluons were emitted by the decay of the parent particle $X$ whose mass is about $\mathcal{O}(100)$ $\mathrm{GeV}-\mathcal{O}(10) \mathrm{TeV}$, they immediately fragment into hadron jets and produce a lot of mesons and baryons $\left(\pi^{ \pm}, \pi^{0}, K^{ \pm}\right.$, $K_{L, S}^{0}, n, p, \Lambda^{0}$, and so on). Then, the typical energy of the produced hadrons is about $\mathcal{O}(1) \mathrm{GeV}-\mathcal{O}(100) \mathrm{GeV}$, and they are injected into the electromagnetic thermal bath which is constituted by $\gamma, e^{ \pm}$, and nucleons.

As we see later, once such high energy hadrons are injected into the thermal bath in the beginning of the $\mathrm{BBN}$ epoch (i.e., at temperature $T \gtrsim 0.09 \mathrm{MeV}$ ), almost all their kinetic energy is transferred into the thermal bath through the electromagnetic interactions except for neutral kaons. Then, the hadrons scatter off the background particles and induce some nonstandard effects on BBN. Extraordinarily, the emitted hadrons interconvert the ambient protons and neutrons with each other through the strong interaction even after the freeze-out time of the neutron to proton ratio $n / p$. For the relatively short lifetime $\left(\tau_{\phi} \simeq 10^{-2} \mathrm{sec}-10^{2} \mathrm{sec}\right)$ in which we are interested, the above effect induces a significant change in the produced light elements. Concretely, protons, which are more abundant than neutrons, are changed into neutrons through the hadron-proton collisions and the ratio $n / p$ increases significantly. In this case, the late-time hadron injection scenario tends to increase ${ }^{4} \mathrm{He}$ because it is the most sensitive to the freeze-out value of $n / p$.

The emitted hadrons do not scatter off the background nucleons directly. At first hadrons scatter off the background photons and electrons because they are much more abundant than background nucleons (about $10^{10}$ times larger). As we see later, for $t \$ 200 \mathrm{sec}$, the emitted high energy hadrons are immediately thermalized through the electromagnetic scattering and reach kinetic equilibrium before they interact with the ambient protons, neutrons, and light elements. Then we use the thermal-averaged cross section $\langle\sigma v\rangle_{N \rightarrow N^{\prime}}^{H_{i}}$ for the strong interaction process $N+H_{i} \rightarrow N^{\prime}+\cdots$ between hadron $H_{i}$ and the ambient nucleon $N$, where $N$ denotes proton $p$ or neutron $n$. The strong interaction rate is estimated by

$$
\begin{aligned}
\Gamma_{N \rightarrow N^{\prime}}^{H_{i}}= & n_{N}\langle\sigma v\rangle_{N \rightarrow N^{\prime}}^{H_{i}} \\
\simeq & \left(2 \times 10^{-8} \mathrm{sec}\right)^{-1} f_{N}\left(\frac{\eta}{10^{-9}}\right)\left(\frac{\langle\sigma v\rangle_{N \rightarrow N^{\prime}}^{H_{i}}}{40 \mathrm{mb}}\right) \\
& \times\left(\frac{T}{1 \mathrm{MeV}}\right)^{3}
\end{aligned}
$$

where $n_{N}$ is the number density of the nucleon species $N, \eta$ is the baryon to photon ratio $\left(=n_{B} / n_{\gamma}\right), n_{B}$ denotes the baryon number density $\left(=n_{p}+n_{n}\right)$, and $f_{N}$ is the nucleon fraction $\left(\equiv n_{N} / n_{B}\right)$. Here, for the moment we adopt $40 \mathrm{mb}$ as a typical value of the cross section for the strong interaction. This equation shows that every hadron whose lifetime is longer than $\mathcal{O}\left(10^{-8}\right)$ sec contributes to the interconverting interaction between neutron and proton at the beginning of BBN. Hereafter we will consider only the long-lived mesons $\left(\pi^{ \pm}, K^{ \pm}\right.$, and $\left.K_{L}\right)$ and baryons $(p, \bar{p}, n$, and $\bar{n}){ }^{3}$ Their lifetimes are given by [27]

$$
\begin{aligned}
\tau_{\pi^{ \pm}} & =(2.6033 \pm 0.0005) \times 10^{-8} \mathrm{sec}, \\
\tau_{K^{ \pm}} & =(1.2386 \pm 0.0024) \times 10^{-8} \mathrm{sec}, \\
\tau_{K_{L}^{0}} & =(5.17 \pm 0.04) \times 10^{-8} \mathrm{sec}, \\
\tau_{n} & =886.7 \pm 1.9 \mathrm{sec},
\end{aligned}
$$

\footnotetext{
${ }^{3} \pi^{0}, K_{S}^{0}$, and $\Lambda^{0}$ have much shorter lifetimes and they have completely decayed because their lifetimes are $\tau_{\pi^{0}}=(8.4 \pm 0.6)$ $\times 10^{-17} \mathrm{sec}, \tau_{K_{S}^{0}}=0.89 \times 10^{-10}$, and $\tau_{\Lambda^{0}}=2.63 \times 10^{-10} \mathrm{sec}$, respectively. Therefore, they do not contribute to the interesting process in this situation.
} 
and the proton is stable.

Here we define the stopping time $\tau_{\text {stop }}^{H_{i}}$ of the high energy particle $H_{i}$ in the thermal plasma as

$$
\tau_{\text {stop }}^{H_{i}}=\int_{E_{0}}^{E_{\mathrm{th}}}\left(\frac{d E}{d t}\right)^{-1} d E,
$$

where $E$ denotes the energy and $d E / d t$ denotes the energy loss rate in the thermal plasma. It also depends on the scattering process of each particle $H_{i}$ off the background particles. $E_{0}$ is the initial energy and $E_{\mathrm{th}}$ is the threshold energy of the process. ${ }^{4}$ To estimate whether particle $H_{i}$ is stopped or not in the thermal plasma through the electromagnetic interaction until it scatters off the background baryons $(n, p$, and produced light elements), we compute the rate,

$$
R_{\text {stop }}^{H_{i}} \equiv \Gamma_{N \rightarrow N^{\prime}}^{H_{i}} \times \tau_{\text {stop }}^{H_{i}}
$$

as an indicator which roughly represents the number of the scattering during the stopping time $\tau_{\text {stop }}^{H_{i}}$. If $R_{\text {stop }}^{H_{i}}$ is much less than unity, the emitted high energy hadron $H_{i}$ is completely stopped and cannot reach the background baryons with the high energy. On the other hand, if $R_{\text {stop }}^{H_{i}}$ is greater than unity, the high energy hadron cannot be stopped through the electromagnetic interaction and directly scatters off the background baryons. In addition, it might destroy the light elements which have already been produced if the particle $X$ decays after the cosmic time is $t \sim 200 \mathrm{sec}$.

\section{B. Hadron stopping in the electromagnetic thermal plasma}

When the cosmic temperature $T$ is higher than the electron mass $m_{e}$, there are sufficient electrons and positrons in the universe. In this situation, it is expected that the emitted charged particles $\pi^{ \pm}, K^{ \pm}$, and $p$ are quickly thermalized through the electromagnetic interaction. In fact, the energy loss rate of the charged particle through the Coulomb scattering is given by

$$
\frac{d E}{d t}=-\frac{\pi}{3} \alpha T^{2}
$$

for $T \gtrsim m_{e}$ in the relativistic regime. $\alpha$ is the fine structure constant $(\simeq 1 / 137)$. Then, the stopping time of the charged particle ("ch") is estimated by

$$
\tau_{\text {stop }}^{\text {ch }} \simeq 1.18 \times 10^{-14} \sec \left(\frac{E}{\mathrm{GeV}}\right)\left(\frac{T}{\mathrm{MeV}}\right)^{-2},
$$

for $T \gtrsim m_{e}$. Then, $R_{\text {stop }}^{\text {ch }}$ is much smaller than unity and we can regard that charged hadrons are completely stopped.

As for the neutron, we can see that it is also completely stopped for $T \gtrsim m_{e}$. Although a neutron is neutral of course, it can scatter off the background electrons through the elec-

\footnotetext{
${ }^{4}$ To roughly estimate the timescale until the particle is stopped, it would be quite adequate that we take $E_{\text {th }}$ to be equal to the mass of the particle $H_{i}$ in the relativistic regime.
}

tromagnetic interaction by the magnetic dipole moment. The energy loss rate through the Coulomb scattering is given by

$$
\frac{d E}{d t}=-\frac{15 m_{n}^{3}}{7 \pi^{3} \alpha^{2} g_{n}^{2} T^{4}},
$$

in the relativistic regime, where $g_{n}$ is the neutron magnetic moment $(=-1.913)$ [27], and $m_{n}$ is neutron mass. The stopping time of a neutron is

$$
\tau_{\text {stop }}^{\mathrm{n}} \simeq 2.34 \times 10^{-10} \sec \left(\frac{T}{\mathrm{MeV}}\right) .
$$

Thus $R_{\text {stop }}^{\mathrm{n}}$ of a neutron is much smaller than unity, and it does not scatter off the background baryons before it stops for $T \gtrsim m_{e}{ }^{5}$

On the other hand, if the temperature is much lower than electron mass $\left(T \lesssim m_{e}\right)$, the situation is quite different because the number density of electrons becomes little. In this case, the emitted mesons completely decay and disappear in the universe before they scatter off the background baryons because the lifetime is shorter than the timescale of the strong interaction [see Eq. (6)]. Thus we should not treat the injection of any mesons in such a late epoch. Because a proton is stable, and a neutron has a long lifetime compared to the typical timescale of the strong interaction in Eq. (6), we should worry about the thermalization of the emitted high-energy nucleons later.

In a proton, for the ionization loss it is more likely to lose the relativistic energy for $T \lesssim m_{e}$. The ionization-loss rate is expressed by

$$
\frac{d E}{d t}=-\frac{Z^{2} \alpha}{v} \omega_{p}^{2} \ln \left(\frac{\Lambda m_{e} \gamma v^{2}}{\omega_{p}}\right),
$$

where $Z$ denotes the charge ( $Z=1$ for proton), $v$ is the velocity of the high energy proton, $\gamma$ is the Lorentz factor, $\Lambda$ is $\mathcal{O}(1)$ constant, and $\omega_{p}$ denotes the plasma frequency

$$
\omega_{p}^{2}=\frac{4 \pi \alpha n_{e}}{m_{e}}
$$

where $n_{e}$ represents the electron number density. We evaluate the stopping time of the proton to lose its relativistic energy,

$$
\tau_{\text {stop }}^{p} \simeq 1.2 \times 10^{-14} \sec x^{1 / 2} e^{x}\left(\frac{E}{\mathrm{GeV}}\right)\left(\frac{\eta_{10}}{5}\right)^{-1}
$$

where $\eta_{10}$ is defined by $\eta=\eta_{10} \times 10^{-10}$, and the dimensionless parameter $x=m_{e} / T$. If we demand $R_{\text {stop }}^{p} \lesssim 1$, we obtain $T \gtrsim 22 \mathrm{keV}$ which corresponds to cosmic time $t \lesssim 3$ $\times 10^{3}$ sec. Namely, after $t \simeq 3 \times 10^{3} \mathrm{sec}$, such a high energy

\footnotetext{
${ }^{5}$ Although the above estimations have been discussed only in the relativistic regime, similar results are also obtained in the nonrelativistic regime [5].
} 
proton cannot be stopped in the thermal bath, and is inevitable to scatter off the ambient baryons with the high energy.

As well as the high energy proton, we estimate the case of the high energy neutron. The energy loss rate of the neutron through the Coulomb scattering for $T \leqq m_{e}$ is

$$
\frac{d E}{d t}=-\frac{3 \pi \alpha^{2} g_{n}^{2} m_{e}}{m_{n}^{2}} n_{e} E^{2} .
$$

The stopping time to lose the relativistic energy is

$$
\tau_{\text {stop }}^{n} \simeq 1.68 \times 10^{-8} \sec x^{3 / 2} e^{x}\left(\frac{E}{\mathrm{GeV}}\right)^{-1}
$$

Here if we require $R_{\text {stop }}^{n} \lesssim 1$, we find that the temperature should be greater than $95 \mathrm{keV}$ for the neutron stopping which corresponds to the condition that cosmic time should be shorter than $150 \mathrm{sec}$. In this case, after $t \simeq 150 \mathrm{sec}$, the high energy neutron will inevitably scatter off the background baryons before it stops. Under these situations, after $t$ $\simeq 150 \mathrm{sec}$ the high energy nucleons scatter off the ambient baryons through the strong interaction; we also have to worry about the possibilities of the destruction of the light elements. This means that the scattering process after $t$ $\simeq 150 \mathrm{sec}$ is beyond the limits of validity in our treatment in this paper. For this problem, we will discuss it later.

As for $K_{L}^{0}$, it is never stopped in the electromagnetic plasma because it does not interact with electrons and photons. Therefore, by using the energy dependent cross sections we will treat the scattering off the ambient nucleons. To perform the computation, we should know the correct energy distribution of $K_{L}^{0}$ produced through the hadron fragmentation.

On the other hand, for relatively longer lifetimes $\tau_{X}$ $\gtrsim 10^{4} \mathrm{sec}$, there is another interesting effect on BBN. The emitted photons or charged leptons induce the electromagnetic cascade showers and produce many soft photons. ${ }^{6}$ Their spectrum has a cutoff at $E_{\gamma}^{\max } \simeq m_{e}^{2} /(22 T)$. If $E_{\gamma}^{\max }$ exceeds the binding energies of the light elements, these photons destroy the light elements and change their abundances $[3,4]$. In fact, at $t \gtrsim 10^{4}\left(10^{6}\right) \mathrm{sec}$, the energy of the photon spectrum which is produced by the decay of $X$ exceeds the deuterium $\left({ }^{4} \mathrm{He}\right)$ binding energy $B_{2} \simeq 2.2\left(B_{4} \simeq 20\right) \mathrm{MeV}$. However, because we are not interested in the photodissociation here, we only study the case of $\tau_{X} \lesssim 10^{4} \mathrm{sec}$. In a separate paper [28], comprehensively we will discuss the effects of both the "hadrodissociation" and the photodissociation of the light elements for longer lifetimes.

\footnotetext{
${ }^{6}$ Even if the decay modes into hadrons are dominant $\left(B_{h}\right.$ $\sim \mathcal{O}(1)$ ), almost all the parts of the energy of the parent particle are transferred into photons and electrons because the hadrons decay after they completely transfer their relativistic energy into the thermal bath. In addition, it is expected that about $1 / 3$ part of the produced hadrons are approximately $\pi^{0}$ and they decay as $\pi^{0} \rightarrow \gamma \gamma$ with a much shorter lifetime $\left(\tau_{\pi^{0}} \simeq 10^{-16} \mathrm{sec}\right)$.
}

\section{Hadron jets and collider experiments}

As an example of the hadronic decay, if the gravitino $\psi_{\mu}$ is the parent particle $X$ whose mass is $m_{X}=\mathcal{O}(1) \mathrm{TeV}$, it can have net hadronic decay modes, e.g., $\psi_{\mu} \rightarrow \tilde{\gamma} q \bar{q}$ ( $q$ : quark), with the branching ratio $B_{h}$. In this case, $B_{h}$ can at least become $\sim \mathcal{O}(\alpha)$ even if the main decay mode is only $\psi_{\mu}$ $\rightarrow \tilde{\gamma} \gamma(\tilde{\gamma}:$ photino $)$, because of the electromagnetic coupling of the photon. As we quantitatively show later, about one hadron is produced for $B_{h}=0.01$ and for the energy per two hadron jets, $2 E_{\text {jet }} \sim 2 / 3 m_{X}$, if we assume that the mechanism of the hadron fragmentation is similar to the $e^{+} e^{-}$collider experiments. In addition, the emitted high energy photon whose energy is about $\sim m_{X} / 2$ scatters off the background photon $\gamma_{\mathrm{BG}}$ and can produce a quark-antiquark pair. ${ }^{7}$ Then, the center of mass energy is about $\sqrt{s} \sim 2 \mathrm{GeV}$ and produces about three hadrons which could effectively contribute to the decay mode into hadrons as the branching ratio $B_{h}$ $\sim \mathcal{O}(0.01)$. Therefore, we should consider the hadronic decay modes at least as $B_{h}=\mathcal{O}(0.01)$ in this case. On the other hand, if the decay mode $\psi_{\mu} \rightarrow \tilde{g} g$ ( $g$ : gluon, and $\tilde{g}$ : gluino) is kinematically allowed, $B_{h}$ may become close to one.

For the other candidate of the parent particle, Polonyi field or moduli, which appears in supergravity or superstring theory and has a $\mathcal{O}(1) \mathrm{TeV}$ mass, would also have a hadronic decay mode $(\phi \rightarrow g g)$.

Fortunately, we can estimate the number and energy distribution of the produced hadrons by using the JETSET 7.4 Monte Carlo event generator [8]. This FORTRAN package computes the hadron fragmentation for the $q \bar{q}$ event $(q$ : quark) in the $e^{+} e^{-}$annihilation and predicts the energy distribution of the products to agree with the $e^{+} e^{-}$collider experiments. In Fig. 2 we plot the averaged charged-particle multiplicity $\left\langle N_{\mathrm{ch}}\right\rangle$ which represents the total number of the charged hadrons emitted per $e^{+} e^{-}$annihilation and per two hadron jets as a function of $\sqrt{s}\left(=2 E_{\text {jet }}\right){ }^{8}$ Recently CERN $e^{+} e-$ collider LEP II experiments (ALEPH, DELPHI, L3, and OPAL) give us the useful data for $\sqrt{s}=130-183 \mathrm{GeV}$. Therefore, now a number of experimental data is available at least up to $\sqrt{s} \simeq 183 \mathrm{GeV}$ [27]. The filled circle denotes the data points of $e^{+} e^{-}$collider experiments. From Fig. 2 we find that the predicted $\left\langle N_{\mathrm{ch}}\right\rangle$ agrees excellently with the experimental values. Thus, in this situation we use the JETSET 7.4 to infer the spectrum of the emitted hadrons extrapolating to the various higher energies.

In Fig. 3 we plot the spectrum of the produced mesons $\left(\pi^{+}+\pi^{-}, K^{+}+K^{-}\right.$, and $\left.K_{L}^{0}\right)$ as a function of the kinetic energy $E_{\text {kin }}$. This is the case that the center of mass energy is

\footnotetext{
${ }^{7}$ Of course, there are some leptonic modes in the process, e.g., $\gamma+\gamma_{\mathrm{BG}} \rightarrow e^{+}+e^{-}$. Thus, the net branching ratio into hadrons is about $\sim 60 \%$ in this energy.

${ }^{8}$ Here $\left\langle N_{\mathrm{ch}}\right\rangle$ is defined as the value after both $K_{S}$ and $\Lambda^{0}$ have completely decayed, where their lifetimes are $\tau_{K_{S}^{0}}=0.89$ $\times 10^{-10} \mathrm{sec}$ and $\tau_{\Lambda^{0}}=2.63 \times 10^{-10} \mathrm{sec}$, respectively. As we have shown in Sec. III A, we should not treat any particles with the shorter lifetime than $\sim 10^{-8} \mathrm{sec}$ in this situation.
} 


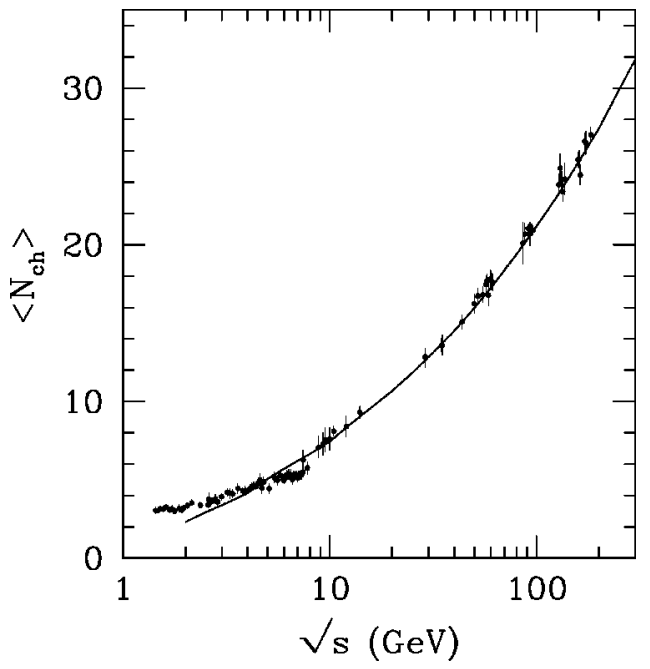

FIG. 2. Plot of the averaged charged-particle multiplicity $\left\langle N_{\mathrm{ch}}\right\rangle$. This represents the total number of the charged hadrons emitted per $e^{+} e^{-}$annihilation and per two hadron jets as a function of $\sqrt{s}$ $\left(=2 E_{\text {jet }}\right)$, where $\sqrt{s}$ denotes the center of mass energy, and $E_{\text {jet }}$ is the energy per one hadron jet. The solid line denotes the value obtained by using the JETSET 7.4 Monte Carlo event generator. The filled circle denotes the data points of $e^{+} e^{-}$collider experiments. Error is quadratically added for the statistical and systematic one. Here $\left\langle N_{\text {ch }}\right\rangle$ is defined as the value after both $K_{S}$ and $\Lambda^{0}$ had completely decayed.

$\sqrt{s}=91.2 \mathrm{GeV}$ which corresponds to the $Z^{0}$ resonance. In similar fashion, in Fig. 4 we plot the spectrum of the produced baryons $[(\mathrm{a}) n+\bar{n}$, and (b) $p+\bar{p}]$. In Fig. 5 we plot the averaged number of the produced hadron per two hadron jets as a function of $2 E_{\text {jet }}$, which is obtained by summing up the energy distribution. From Fig. 5, we find that almost all hadrons are composed of pions.

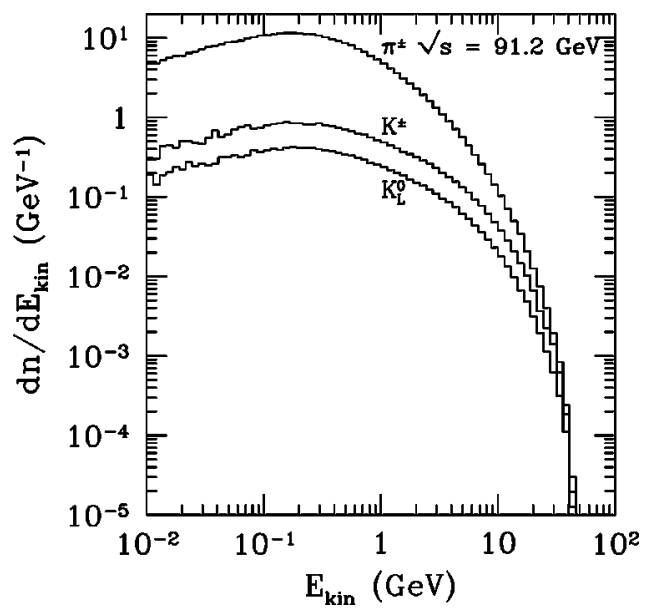

FIG. 3. Plot of the spectrum of the produced mesons $\left(\pi^{+}\right.$ $+\pi^{-}, K^{+}+K^{-}$, and $K_{L}^{0}$ ) as a function of the kinetic energy $E_{\text {kin }}$. This is the case that the center of mass energy is $\sqrt{s}=91.2 \mathrm{GeV}$ which corresponds to the $Z^{0}$ resonance. They are computed by using the JETSET 7.4 Monte Carlo event generator.

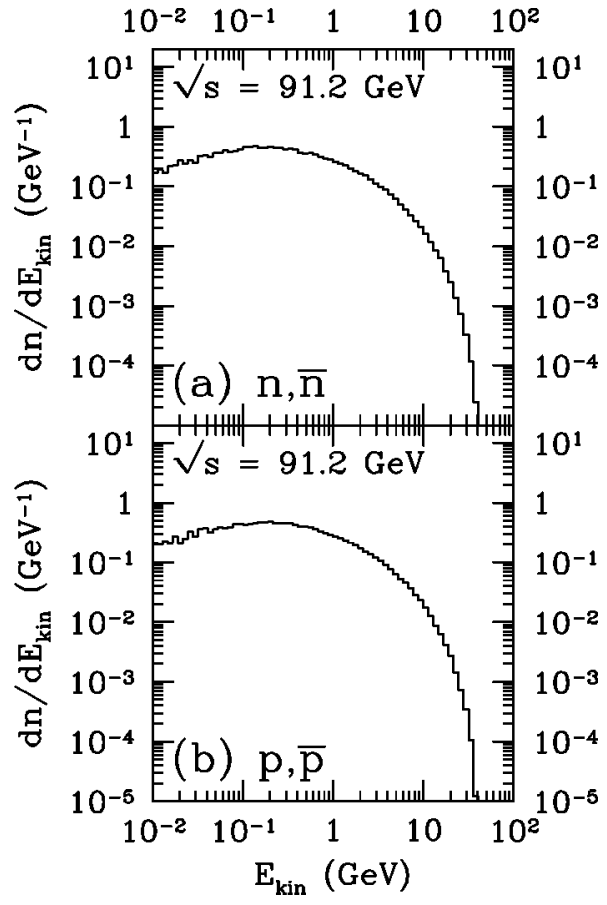

FIG. 4. Plot of the spectrum of the produced baryons [(a) $n$ $+\bar{n}$ and (b) $p+\bar{p}]$ as a function of the kinetic energy $E_{\mathrm{kin}}$. This is the case that the center of mass energy is $\sqrt{s}=91.2 \mathrm{GeV}$ which corresponds to the $Z^{0}$ resonance. They are computed by using the JETSET 7.4 Monte Carlo event generator.

\section{Cross sections of hadron-nucleon scattering}

Because in this paper we are interested in the BBN epoch, i.e., $T \lesssim \mathcal{O}(1) \mathrm{MeV}$, the temperature is much less than the

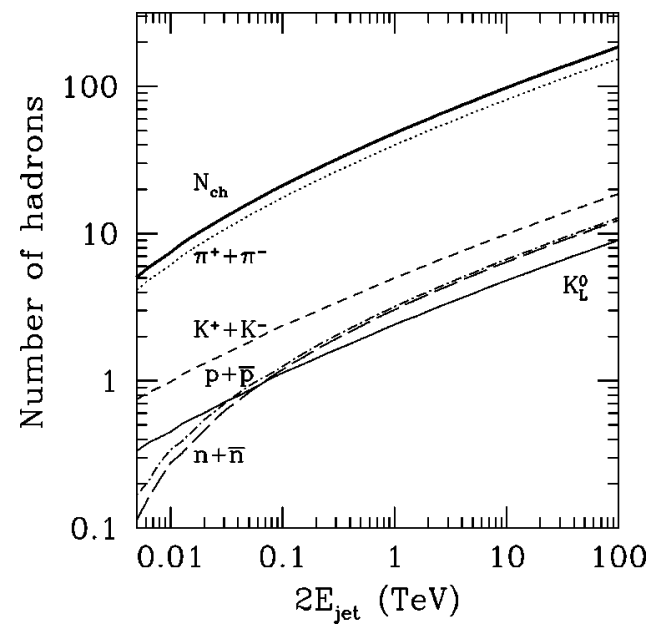

FIG. 5. Plot of the averaged number of the produced hadrons as a function of $2 E_{\text {jet }}(=\sqrt{s})$, where $E_{\text {jet }}$ denotes the energy of one hadron jet. The number is defined by the value per two hadron jets. $\left\langle N_{\mathrm{ch}}\right\rangle$ denotes the averaged charged-particle multiplicity (thick solid line). The number is obtained by summing up the energy distribution. The dotted line is $\pi^{+}+\pi^{-}$, the short dashed line is $K^{+}$ $+K^{-}$, the thin solid line is $K_{L}^{0}$, the dot-dashed line is $p+\bar{p}$, and the long dashed line is $n+\bar{n}$. They are computed by using the JETSET 7.4 Monte Carlo event generator. 
typical mass of the emitted hadrons, e.g., $m_{H_{i}}$ $=\mathcal{O}(100) \mathrm{MeV}-\mathcal{O}(1) \mathrm{GeV}$. As we discussed in Sec. III B, as long as the temperature is relatively high enough ( $T$ $\gtrsim 95 \mathrm{keV})$, the emitted high energy hadrons $\left(\pi^{ \pm}, K^{ \pm}, p\right.$, and $n$ ) have completely lost their relativistic energies through the electromagnetic interaction in the thermal plasma and are quickly thermalized except for neutral kaon $K_{L}^{0}$. Then only the exothermic process is relevant for the hadron to scatter off the background baryons through the strong interaction because it has just a little kinetic energy of the order of the temperature $T$. Of course, such a low energy hadron cannot destroy the background ${ }^{4} \mathrm{He}$. Concerning exothermic reactions, it is well-known that the cross section $\sigma$ is nearly inversely proportional to the velocity $v$ of the projectile particle in the low energy. Namely, $\sigma v$ almost does not have a $v$ dependence and is nearly a constant for the beam energy. Except for $K_{L}^{0}$, we can use the threshold cross section instead of the thermal-averaged cross section. Here we adopt the results of the thermal-averaged cross section in Ref. [5].

The thermally averaged cross sections for $\pi^{ \pm}$are

$$
\begin{aligned}
& \langle\sigma v\rangle_{n \rightarrow p}^{\pi^{+}}=1.7 \mathrm{mb}, \\
& \langle\sigma v\rangle_{p \rightarrow n}^{\pi^{-}}=1.5 C_{\pi}(T) \mathrm{mb},
\end{aligned}
$$

where $C_{H_{i}}(T)$ is the Coulomb correction factor when the beam particle $H_{i}$ is the charged one. Because the reaction $p^{+}+\pi^{-} \rightarrow n+\cdots$ is enhanced due to the opposite-sign charge of the initial state particles, we should correct the strong interaction rates by simply multiplying $C_{H_{i}}(T)$ to that which is obtained by ignoring the Coulomb corrections. The Coulomb correction factor is generally estimated by

$$
C_{H_{i}}(T)=\frac{2 \pi \xi_{i}(T)}{1-e^{-2 \pi \xi_{i}(T)}},
$$

where $\xi_{i}(T)=\alpha \sqrt{\mu_{i} / 2 T}, \alpha$ is the fine structure constant, and $\mu_{i}$ is the reduced mass of the hadron $H_{i}$ and the nucleon.

The thermally-averaged cross sections for $K^{-}$are

$$
\begin{aligned}
& \langle\sigma v\rangle_{n \rightarrow p}^{K^{-}}=26 \mathrm{mb}, \\
& \langle\sigma v\rangle_{n \rightarrow n}^{K^{-}}=34 \mathrm{mb}, \\
& \langle\sigma v\rangle_{p \rightarrow n}^{K^{-}}=31 C_{K}(T) \mathrm{mb}, \\
& \langle\sigma v\rangle_{p \rightarrow p}^{K^{-}}=14.5 C_{K}(T) \mathrm{mb} .
\end{aligned}
$$

Here we ignore $K^{+}$interaction because $n+K^{+} \rightarrow p+K^{0}$ is the endothermic reaction which has $Q=2.8 \mathrm{MeV}$, and it is expected that the kinetic energy of $K^{+}$is less than $Q$.

As for neutral kaon $K_{L}^{0}$, there are no adequate experimental data of the differential cross sections as a function of the beam energy to use in our current purpose. It is very serious for us because $K_{L}^{0}$ does not lose its relativistic energy and is never stopped in the thermal bath. We should then know the

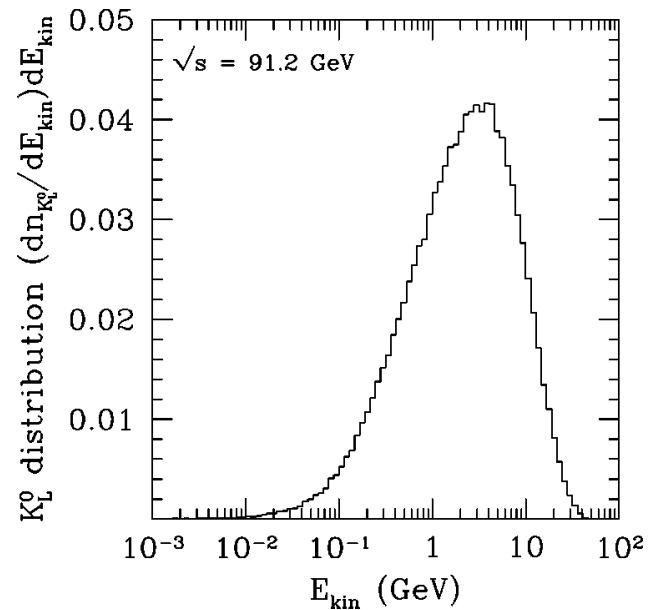

FIG. 6. Plot of the distribution of $K_{L}^{0}$ produced in the $e^{+} e^{-}$ annihilation as a function of the kinetic energy. It is the case that the center of mass energy is $\sqrt{s}=91.2 \mathrm{GeV}$ which corresponds to the $Z^{0}$ resonance. It is computed by the JETSET 7.4 Monte Carlo event generator.

differential cross sections in the whole relevant energy range. For example, in Fig. 6 we find that the source distribution function of $K_{L}^{0}$ is spread in the wide energy range. At least we want to obtain the data of the cross sections for the typical $K_{L}^{0}$-beam energy, $E_{\text {beam }}=10 \mathrm{MeV}-1 \mathrm{TeV}$, where $E_{\text {beam }}$ is the kinetic energy of $K_{L}^{0}$. In this situation, we should estimate the data table of the cross sections of the $K_{L}^{0}$ scattering by using the other existing experimental information.

Here we assume that $K_{L}^{0}$ scatters off the nucleon $N$ as a combination of $1 / 2 K^{0}$ and $1 / 2 \bar{K}^{0}$ because in fact $K_{L}^{0}$ is nearly the linear combination of $K^{0}$ and $\bar{K}^{0}$ states that $\left|K_{L}^{0}\right\rangle$ $\simeq\left(\left|K^{0}\right\rangle-\left|\bar{K}^{0}\right\rangle\right) / \sqrt{2}{ }^{9}{ }^{9}$ In addition, we assume that the strangeness of $K^{0}\left(\bar{K}^{0}\right)$ is similar to $K^{+}\left(K^{-}\right)$because $K^{0}$ $=d \bar{s}\left(\bar{K}^{0}=s \bar{d}\right)$ contains $\bar{s}(s)(s$ : strange quark, and $d$ : down quark). Of course, the above assumption is not wrong very much under the isospin $\mathrm{SU}(2)$ transformation for the $\left(\begin{array}{l}u \\ d\end{array}\right)$ doublet ( $u$ : up quark) because we cannot imagine that there exists a special coherent interference in the inelastic scattering.

In this assumption, we would also have to worry about the effect of the Coulomb corrections because $K^{0} N$ scatterings are not supposed to suffer from any electric charges. From Eq. (24) however, we find that the Coulomb correction is less than $10 \%$ at most in both cases of the attractive force and the repulsive one as long as the kinetic energy of $K^{ \pm}$is more than $\mathcal{O}(10) \mathrm{MeV}$. Therefore, we can ignore the Coulomb correction and the above assumption would be reasonable in this situation.

Fortunately, we have good compilations of the experiments for the total cross section and the elastic cross section for $K^{+} p$ and $K^{-} p$ [27]. Thus, by averaging them we can

\footnotetext{
${ }^{9}$ Of course, the $C P$ violation effect does not change our rough estimates at all and is not important here.
} 


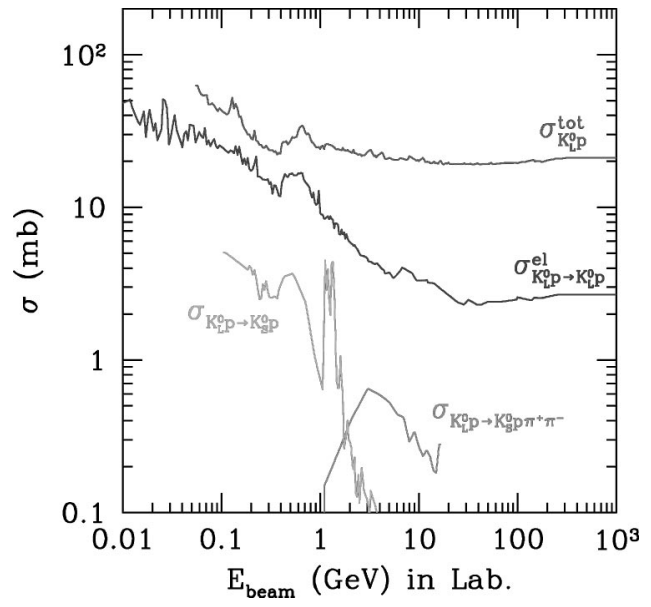

FIG. 7. Plot of the data of the cross sections as a function of the kinetic energy of the $K_{L}^{0}$ beam.

estimate the total $\sigma_{K^{0} p}^{\mathrm{tot}}$ and elastic cross section $\sigma_{K_{L}^{0} p}^{\mathrm{el}}$, respectively. In Fig. 7, we plot the obtained total and elastic cross sections for $K_{L}^{0} p$ scattering. It is fortunate that the obtained total cross sections agree with the direct experimental data and the theoretical predictions marginally within a few tens percent although they were studied only in the low energy regions for $E_{\text {beam }} \leq 0.7 \mathrm{GeV}$ [29]. In addition, we have the experimental data of the inelastic scatterings, $K_{L}^{0} p$ $\rightarrow K_{S}^{0} p$ and $K_{L}^{0} p \rightarrow K_{S}^{0} p \pi^{+} \pi^{-}$[30] which are also plotted in Fig. 7. Now we assume that the cross section of the interconverting reaction $K_{L}^{0}+p \rightarrow n+\cdots$ is obtained by $\sigma_{p \rightarrow n}^{K_{L}^{0}}$ $=1 / 2\left[\sigma_{K_{L}^{0} p}^{\mathrm{tot}}-\left(\sigma_{K_{L}^{0} p \rightarrow K_{L}^{0} p}^{\mathrm{el}}+\sigma_{K_{L}^{0} p \rightarrow K_{S}^{0} p}+\sigma_{K_{L}^{0} p \rightarrow K_{S}^{0} p \pi^{+} \pi^{-}}\right)\right]$because the final states of the inelastic scattering without $K_{L}^{0} p$ $\rightarrow K_{S}^{0} p+\cdots$ are $K N \pi, \Lambda^{0} \pi$, or $\Sigma \pi$, and it is approximately expected that either $p$ or $n$ appears in a closely even probability. ${ }^{10}$ Then, we get the remaining cross section as $\sigma_{p \rightarrow p}^{K_{L}^{0}}=\sigma_{K_{L}^{0} p}^{\text {tot }}-\sigma_{p \rightarrow n}^{K_{L}^{0}}$.

About neutron- $K_{L}^{0}$ scattering, we could have performed similar treatments. However, compared to the cases of a proton, we do not have adequate compilations for the neutron$K^{ \pm}$process. On the other hand, the data tell us that we can approximately regard them as the cross sections of the proton- $K$ scattering within a few tens percent in the high beam energies $\left(E_{K} \gtrsim 100 \mathrm{MeV}\right)$. The theoretical reason is that the strong interaction does not distinguish between a proton and a neutron in such a high energy. Under these circumstances, we assume that the cross section of $K_{L}^{0} n$ is the same as $K_{L}^{0} p$ with a few tens percent error.

To perform the numerical computations including the $K_{L}^{0}$-injection effects in BBN, it is useful to average the cross

\footnotetext{
${ }^{10}$ The branching ratios are presented as $\Lambda^{0} \rightarrow n \pi^{0}(35 \%)$, $p \pi^{-}(63.9 \%) ; \quad \Sigma^{0} \rightarrow \Lambda^{0} \gamma(100 \%) ; \quad \Sigma^{+} \rightarrow n \pi^{+}(48.3 \%)$, $p \pi^{0}(51.6 \%) ; \Sigma^{-} \rightarrow n \pi^{-}(99.9 \%)$ [27]. We also ignore the multiple production process of baryons because the center of mass energy is too low for the process to dominate the other reactions.
}

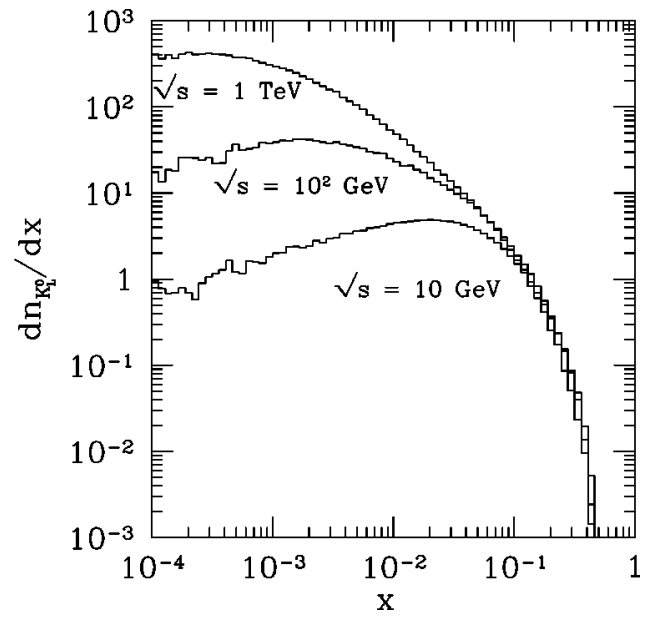

FIG. 8. Plot of the spectrum of the $K_{L}^{0}$ produced through the hadron fragmentation of $q \bar{q}$ pair emitted from $e^{+} e^{-}$annihilation. $x$ $\left(\equiv E_{\text {kin }} / \sqrt{s}\right)$ denotes the normalized kinetic energy $E_{\text {kin }}$ and $\sqrt{s}$ denotes the center of mass energy of $e^{+} e^{-}$collision. They are computed by using the JETSET 7.4 Monte Carlo event generator.

sections by the energy spectrum of $K_{L}^{0}$. As we discussed in the previous subsection, we can use the JETSET 7.4 Monte Carlo generator and get the energy spectrum of emitted $K_{L}^{0}$ in wide range of the source energy. For example, we can see the spectrum of the produced $K_{L}^{0}$ for various energies in Fig. 8 . Then, we get the averaged cross sections, $\bar{\sigma}_{p \rightarrow p}^{K_{L}}$ and $\bar{\sigma}_{p \rightarrow n}^{0}$, as the convolutions of the data of the cross sections with the energy spectrum of $K_{L}^{0}$ (Fig. 9).

Concerning the emitted nucleons, we basically follow Reno and Seckel's treatment that we regard the nucleonantinucleon pair as a kind of a meson $N \bar{N}$ [5]. Then, the $N \bar{N}$ meson induces the interconversion $N+N \bar{N} \rightarrow N^{\prime}+\cdots$. In Ref. [5], we have the thermally-averaged cross sections

$$
\langle\sigma v\rangle_{n \rightarrow n}^{n \bar{n}}=37 \mathrm{mb}
$$

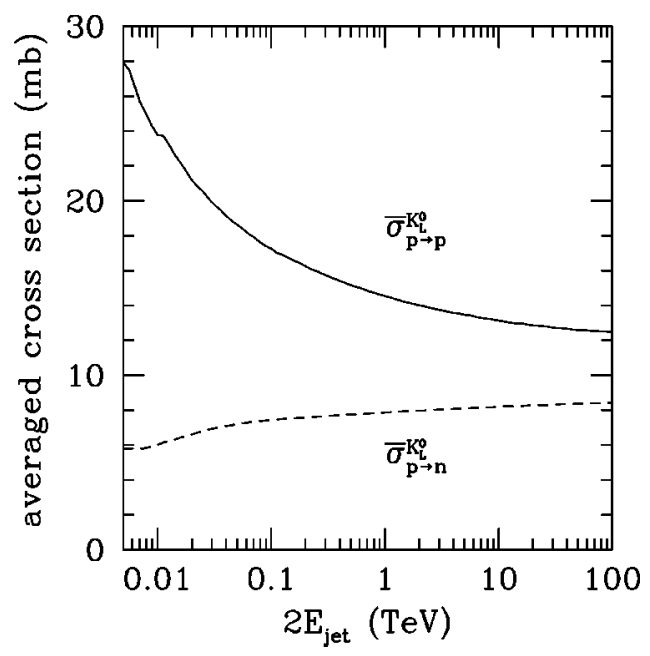

FIG. 9. Plot of the averaged cross sections for $p+K_{L}^{0} \rightarrow p$ $+\cdots$ and $p+K_{L}^{0} \rightarrow n+\cdots$ as a function of the energy of two jets $\left(=2 E_{\text {jet }}\right)$. 


$$
\begin{aligned}
& \langle\sigma v\rangle_{p \rightarrow n}^{n \bar{n}}=28 \mathrm{mb}, \\
& \langle\sigma v\rangle_{n \rightarrow p}^{p \bar{p}}=28 \mathrm{mb}, \\
& \langle\sigma v\rangle_{p \rightarrow p}^{p \bar{p}}=37 \mathrm{mb} .
\end{aligned}
$$

As we discussed in the previous sections, however, the late time emission of the high energy nucleons would induce the destruction of light elements for $T \$ 95 \mathrm{keV}$. However, for the moment we treat the nucleons as if they are approximately thermalized. We will also discuss the modification of the result caused by the above simple assumption later.

\section{E. Formulation in hadron-injection scenario}

We formulate the time evolution equations in the late-time hadron-injection scenario here. As we have mentioned in the previous subsections, the hadron injection at the beginning of BBN enhances the interconverting interactions between neutron and proton equally and the freeze-out value of $n / p$ is extremely increased. Then the time evolution equations for the number density of a nucleon $N(=p, n)$ is represented by

$$
\frac{d n_{N}}{d t}+3 H(t) n_{N}=\left[\frac{d n_{N}}{d t}\right]_{\text {weak }}-B_{h} \Gamma_{X} n_{X}\left(K_{N \rightarrow N^{\prime}}-K_{N^{\prime} \rightarrow N}\right),
$$

where $H(t)$ is the Hubble expansion rate, $\left[d n_{N} / d t\right]_{\text {weak }}$ denotes the contribution from the usual weak interaction rates as well as $\mathrm{SBBN}, B_{h}$ is the branching ratio of the hadronic decay mode of $X, n_{X}$ is the number density of $X, K_{N \rightarrow N^{\prime}}$ denotes the average number of the transition $N \rightarrow N^{\prime}$ per one $X$ decay.

The average number of the transition $N \rightarrow N^{\prime}$ per one $X$ decay is expressed by

$$
K_{N \rightarrow N^{\prime}}=\sum_{H_{i}} \frac{N_{\text {jet }}}{2} N^{H_{i}} R_{N \rightarrow N^{\prime}}^{H_{i}},
$$

where $H_{i}$ runs the hadron species which are relevant to the nucleon interconverting reactions, $N_{\text {jet }}$ is the number of the hadron jet per one $\mathrm{X}$ decay, $N^{H_{i}}$ denotes the average number of the emitted hadron species $H_{i}$ per one $X$ decay. $N^{H_{i}}$ is presented in Fig. 5 as a function of $2 E_{\text {jet }}$, where $E_{\text {jet }}$ is the energy of a hadron jet. $R_{N \rightarrow N^{\prime}}^{H_{i}}$ denotes the probability that a hadron species $H_{i}$ induces the nucleon transition $N \rightarrow N^{\prime}$ and is represented by

$$
R_{N \rightarrow N^{\prime}}^{H_{i}}=\frac{\Gamma_{N \rightarrow N^{\prime}}^{H_{i}}}{\Gamma_{\mathrm{dec}}^{H_{i}}+\Gamma_{\mathrm{abs}}^{H_{i}}}
$$

where $\Gamma_{\text {dec }}^{H_{i}}=\tau_{H_{i}}^{-1}$ is the decay rate of $H_{i}, \tau_{H_{i}}$ is the lifetime, and $\Gamma_{\text {abs }}^{H_{i}} \equiv \Gamma_{N \rightarrow N^{\prime}}^{H_{i}}+\Gamma_{N^{\prime} \rightarrow N}^{H_{i}}+\Gamma_{N \rightarrow N}^{H_{i}}+\Gamma_{N^{\prime} \rightarrow N^{\prime}}^{H_{i}}$ is the total absorption rate of $H_{i}$.

Because the emitted high energy $K_{L}^{0}$ is not stopped in the thermal bath, its lifetime becomes longer by a factor of $E_{K_{L}^{0}} / m_{K_{L}^{0}}$ due to the relativistic effect. Then, the decay rate is

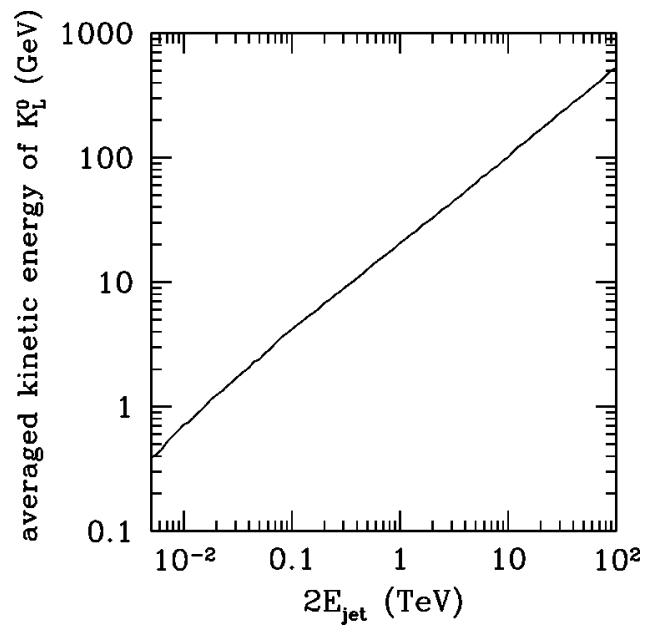

FIG. 10. Plot of the mean kinetic energy of $K_{L}^{0}$ which is obtained by weighting the kinetic energies for their distribution as a function of $2 E_{\text {jet }}$, where $2 E_{\text {jet }}$ is the energy of two hadron jets.

estimated by $\Gamma_{\mathrm{dec}}^{K_{L}^{0}}=\tau_{K_{L}^{0}}^{-1} m_{K_{L}^{0}} / E_{K_{L}^{0}}$. Because the emitted $K_{L}^{0}$, s are distributed in the wide energy range, for convenience we compute the mean kinetic energy $\bar{E}_{K_{L}^{0}}$ which is obtained by weighting the kinetic energies for their distribution (see Fig. 8). In Fig. 10, $\bar{E}_{K_{L}^{0}}$ is plotted as a function of $2 E_{\text {jet }}$.

\section{BBN COMPUTATION IN HADRON-INJECTION SCENARIO AND COMPARISON WITH OBSERVATIONS}

In this section we perform the $\mathrm{BBN}$ computations in the hadron-injection scenario. Then we compare the theoretical prediction of the light element abundances with the observational light element abundances. In the computations we assume that the massive particle $X$ decays into three bodies $\left(E_{\text {jet }}=m_{X} / 3\right)$ and two jets are produced at the parton level (i.e., the number of jets $N_{\text {jet }}=2$ ). The above choice of a set of model parameters $E_{\text {jet }}$ and $N_{\text {jet }}$ is not unique in general and is obviously model dependent. For $E_{\text {jet }}$ however, since we study the wide range of the mass, we can read off the results by rescaling the mass parameter. In addition, for the modification of $N_{\text {jet }}$ since the second term in the right hand side in Eq. (33) scales as $\propto N_{\text {jet }}$, we only translate the obtained results according to the above scaling rule and push the responsibility off onto the number density $n_{X}$.

As we noted in the previous sections, it is a remarkable feature that the predicted ${ }^{4} \mathrm{He}$ mass fraction $Y$ tends to increase in the hadron injection scenario because ${ }^{4} \mathrm{He}$ is the most sensitive to the freeze-out value of the neutron to proton ratio in the beginning of BBN. Since protons which are more abundant than neutrons are changed into neutrons through the strong interactions rapidly, the freeze-out value of $n / p$ increases significantly once the net hadrons are emitted. In addition, $\mathrm{D}$ is also sensitive to the neutron number after $T \leqq 0.1 \mathrm{MeV}$ because the free neutrons cannot burn into ${ }^{4} \mathrm{He}$.

To see the rough tendency, we plot the upper bounds for $B_{h} n_{X} / s$ in Fig. 11 which come from each observational $2 \sigma$ 


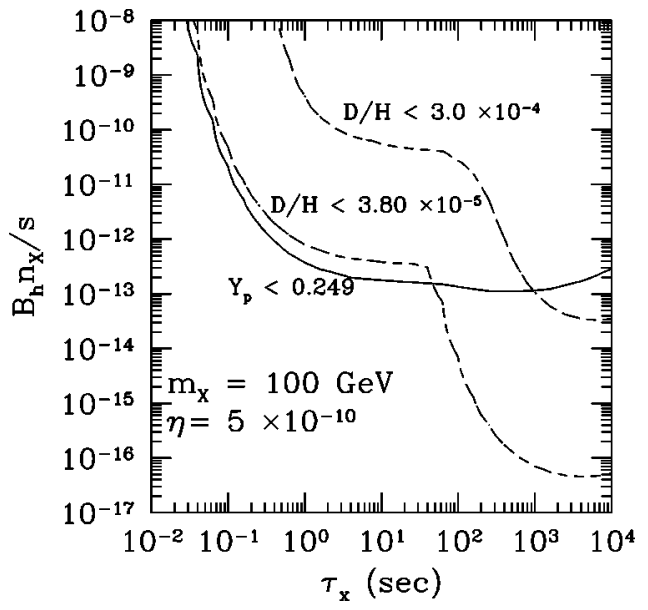

FIG. 11. Plot of the rough upper bound of $B_{h} n_{X} / s$ from the observational $2 \sigma$ upper bounds of ${ }^{4} \mathrm{He}$ (solid line), and D (dashed line) for high $\mathrm{D}$ or low $\mathrm{D}$ as a function of the lifetime of the massive particle $X . B_{h}$ is the hadronic branching ratio of $X$, and $n_{X} / s$ denotes the number density of $X$ per entropy density $s$. Here the baryon to photon ratio is $\eta=5 \times 10^{-10}$ and the mass of $X\left(m_{X}\right)$ is fixed to be $100 \mathrm{GeV}$. The observational upper bounds are obtained by adding the errors in quadrature.

upper bound for ${ }^{4} \mathrm{He}$, and $\mathrm{D}$ as a function of the lifetime $\tau_{X}$ at the baryon to photon ratio $\eta=5 \times 10^{-10} \cdot{ }^{11} B_{h}$ is the hadronic branching ratio of $X$, and $n_{X} / s$ denotes the number density of $X$ per entropy density $s$. The mass is fixed to be typical value, $m_{X}=100 \mathrm{GeV}$. From the figure, we find that for the shorter lifetime $\tau_{X} \lesssim 10^{-2} \mathrm{sec}$, the hadron injections do not affect the freeze-out value of $n / p$ and do not change any predictions of SBBN. However, if the lifetime becomes longer $\tau_{X} \geq 10^{-2} \mathrm{sec}$, the freeze-out value of $n / p$ ratio is increased by the hadron-induced interconverting interactions and the produced neutron increases the ${ }^{4} \mathrm{He}$ abundance, because most of the free neutrons burn into ${ }^{4} \mathrm{He}$ through D. Then, $n_{X} / s$ is strongly constrained by the upper bound of the observational ${ }^{4} \mathrm{He}$ abundance. For $\tau_{X} \gtrsim 10^{2} \mathrm{sec}$, since the produced free $\mathrm{D}$ can no longer burn into ${ }^{4} \mathrm{He}$, the extra free neutrons still remain in $\mathrm{D}$. Then $n_{X} / s$ is severely constrained by the upper bound of the observational $\mathrm{D} / \mathrm{H}$. For the constraint from high $\mathrm{D}$, i.e., $\mathrm{D} / \mathrm{H}<3.0 \times 10^{-4}$, we obtain the milder upper bound than low $\mathrm{D}$ because more productions are allowed from the observation.

However, you can easily find that these constraints are obtained only when $\eta$ is fixed. If we chose the other $\eta$, e.g., which predicts more $\mathrm{D} / \mathrm{H}$ than the upper bound of the observation in SBBN, then, almost all the parameter regions would have been excluded because both $\mathrm{D}$ and ${ }^{4} \mathrm{He}$ tend to increase in the hadron-injection scenario. Namely, any constraints, which are obtained when we fix $\eta$ a priori, have

\footnotetext{
${ }^{11} \mathrm{The}{ }^{7} \mathrm{Li}$ abundance is mildly constrained from the observation and is much weaker than the others. In addition since it has a complicated dependence for $\eta$, we do not plot it here. Of course, however, we include ${ }^{7} \mathrm{Li}$ in Monte Carlo simulation and maximum likelihood analysis which will be discussed below.
}

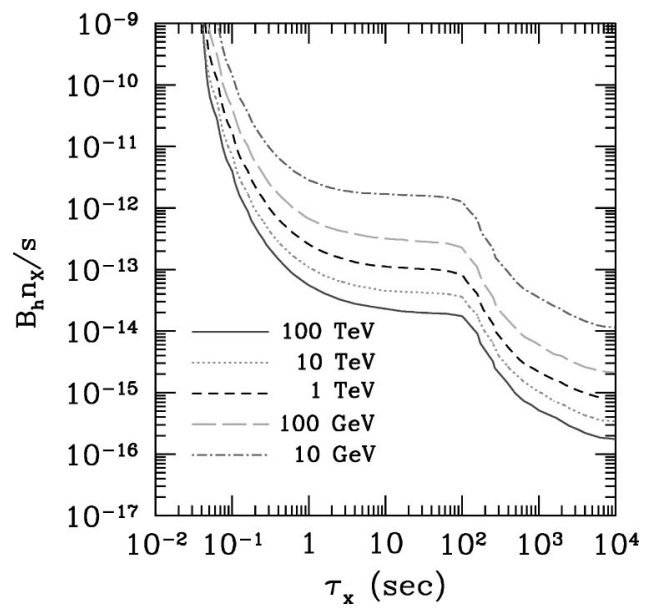

FIG. 12. Plot of the contour of the confidence level (C.L.) in $\left(\tau_{X}, B_{h} n_{X} / s\right)$ plane for low $\mathrm{D}$. The region below the line is allowed by the observations at $95 \%$ C.L. $\tau_{X}$ is the lifetime of $X, B_{h}$ is the branching ratio into hadrons, and $n_{X} / s$ denotes the number density of $X$ per entropy density. It is the case that the mass of $X$ is $m_{X}$ $=100 \mathrm{TeV}$ (solid line), $10 \mathrm{TeV}$ (dotted line), $1 \mathrm{TeV}$ (dashed line), $100 \mathrm{GeV}$ (long dashed line), or $10 \mathrm{GeV}$ (dot-dashed line), respectively.

little meaning. To correctly compare each model in the various parameters $\left(\eta, \tau_{X}\right.$, and $\left.n_{X} / s\right)$, we should perform the maximum likelihood analysis and the $\chi^{2}$ fitting in wide parameter region including both the observational and theoretical errors. To estimate the theoretical errors, we perform the Monte Carlo simulation including the theoretical uncertainties which come from experimental errors of nuclear reaction and hadron-nucleon reaction rates.

Concerning the detail of the executions, we have already explained in Sec. II B. For the hadron-nucleon interaction rate, we adopt 50\% error for each cross section because there are not any adequate experimental data for the uncertainties of cross sections. Therefore, we take the larger errors to get a conservative bound here.

In Fig. 12 we plot the results of the $\chi^{2}$ fitting at 95\% C.L. in $\left(\tau_{X}, B_{h} n_{X} / s\right)$ plane projected on $\eta$ axis in the case of low $\mathrm{D}$ which is obtained by performing the maximum likelihood analysis. The region below the line is allowed by the observations $\left({ }^{4} \mathrm{He}, \mathrm{D}\right.$, and $\left.{ }^{7} \mathrm{Li}\right)$ for the various mass of $X$. If $m_{X}$ becomes heavier, more hadrons are emitted in the decay, and the upper bound becomes more stringent. Comparing the case of $m_{X}=100 \mathrm{GeV}$ in Fig. 12 with that in Fig. 11, the upper bound obtained in the Monte Carlo simulation is milder. That is because we did not adopt the naive $2 \sigma$ observational upper bounds with fixed $\eta$, but we searched the wide range of $\eta$, not forgetting $\tau_{X}$ and $n_{X} / s$, and we performed the maximum likelihood analysis to include both all the observational and theoretical uncertainties. In Fig. 13 we also plot the results of high D. Compared to the case of low D (Fig. 12), the obtained upper bound becomes milder because more $\mathrm{D}$ is allowed by the observations in the high $\mathrm{D}$ case.

As we also discussed in the previous section, the above treatment might underestimate the deuterium abundance for $\tau_{X} \gtrsim 150 \mathrm{sec}$ because deuterium is produced by the destruc- 


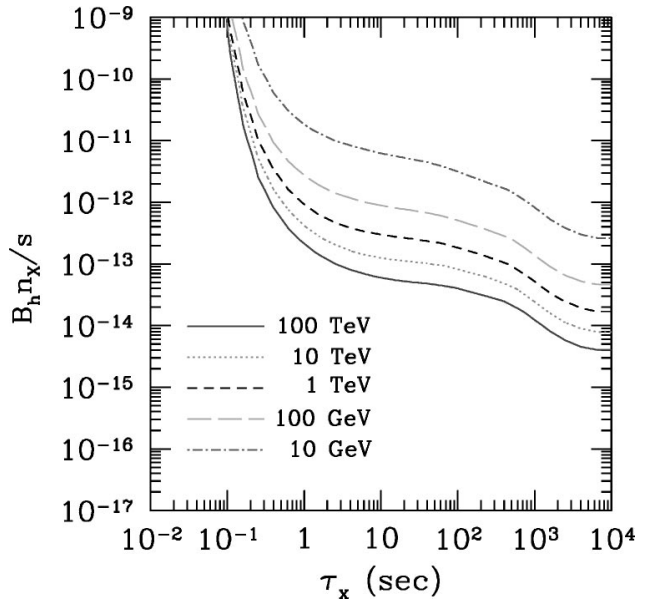

FIG. 13. Plot of the contour of the confidence level (C.L.) in $\left(\tau_{X}, B_{h} n_{X} / s\right)$ plane for high $\mathrm{D}$. The region below the line is allowed by the observations at $95 \%$ C.L. $\tau_{X}$ is the lifetime of $X, B_{h}$ is the branching ratio into hadrons, and $n_{X} / s$ denotes the number density of $X$ per entropy density. It is the case of the mass $m_{X}$ $=100 \mathrm{TeV}$ (solid line), $10 \mathrm{TeV}$ (dotted line), $1 \mathrm{TeV}$ (dashed line), $100 \mathrm{GeV}$ (long dashed line), or $10 \mathrm{GeV}$ (dot-dashed line), respectively.

tion of ${ }^{4} \mathrm{He}$ by the high-energy free neutrons in such a relatively late epoch. Now in a separate paper [28] we are studying the problem of "hadrodissociation" effects. Therefore, that means we obtained the conservative limits only for a longer lifetime than $150 \mathrm{sec}$ in this paper.

Here we consider one of the concrete models of the hadronic decay. If we assume that the parent massive particle is gravitino and that it mainly decays into a photon and a photino $\left(\psi_{3 / 2} \rightarrow \tilde{\gamma}+\gamma\right)$, the lifetime $\tau_{3 / 2}$ is related to the gravitino mass $m_{3 / 2}$ as

$$
\tau_{3 / 2} \simeq 4 \times 10^{2} \sec \times\left(\frac{m_{3 / 2}}{10 \mathrm{TeV}}\right)^{-3}
$$

In addition, if we assume that the gravitino is produced through the thermal scattering in the reheating process after inflation, ${ }^{12}$ we relate the abundance $n_{3 / 2} / s$ of the gravitino with the reheating temperature $T_{R}[2]$,

$$
\frac{n_{3 / 2}}{s} \simeq 1.6 \times 10^{-12} \times\left(\frac{T_{R}}{10^{10} \mathrm{GeV}}\right) .
$$

\footnotetext{
${ }^{12}$ Recently it was claimed that gravitinos are also produced in the preheating epoch nonthermally [31-33]. However, we do not consider such processes here because there are some ambiguities on the estimations and they depend on various model parameters. In addition, very recently it was pointed out that such an effect is not important if we realistically consider two chiral multiplets to distinguish between inflatino and goldstino [34]. If the nonthermal production is effective, however, the obtained constraint might be more severe.
}

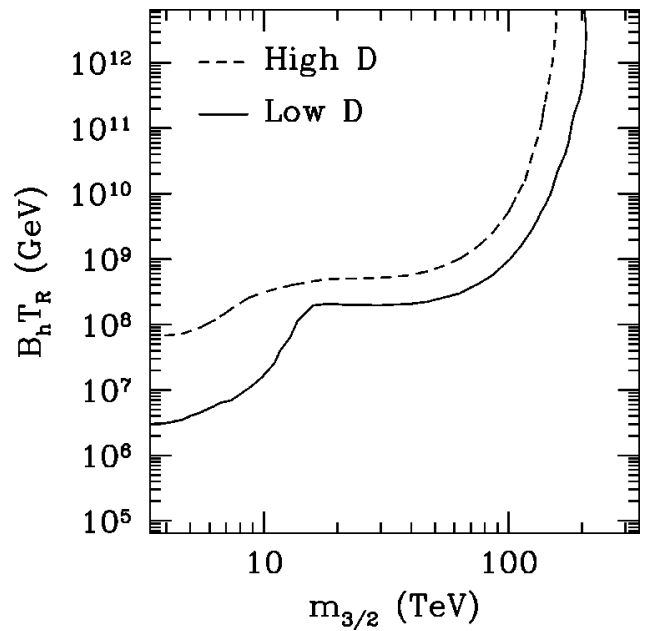

FIG. 14. Plot of the upper bound on the reheating temperature after inflation at $95 \%$ C.L. as a function of the gravitino mass $m_{3 / 2}$. Here $B_{h}$ is the branching ratio into hadrons $(=0.01-1)$. The solid line (dashed line) denotes the case of low D (high D). The region below the line is allowed by the observations.

In Fig. 14 we plot the upper bound on the reheating temperature after inflation at $95 \%$ C.L. as a function of the gravitino mass $m_{3 / 2}$. The solid line (dashed line) denotes the case of low $\mathrm{D}$ (high $\mathrm{D}$ ). The region below the line is allowed by the observations. As we discussed before, $B_{h}$ can at least become $\sim \mathcal{O}(\alpha)$ even if the main decay mode is only photons, because photons have the electromagnetic coupling with $q \bar{q}$, i.e., $\left(B_{h}=0.01-1\right)$. For $m_{3 / 2} \lesssim 10 \mathrm{TeV}$, they mean the conservative upper bound.

\section{SUMMARY AND CONCLUSIONS}

In this paper we have discussed the effects of the late-time hadron injection on the primordial nucleosynthesis which are caused by the decay of an unstable massive particle $X$ when the lifetime is relatively short, $10^{-2} \mathrm{sec} \leq \tau_{X} \leq 10^{4} \mathrm{sec}$. If the massive particle decays into quarks or gluons, they quickly fragment into hadrons. Then the high energy hadrons would be emitted into the electromagnetic thermal bath near the BBN epoch. Because the background photons and electrons are sufficiently energetic in the epoch, such high energy hadrons lose almost all their kinetic energies through the electromagnetic interaction, and they are approximately stopped before they interact with the background nucleons ( $p$ and $n$ ) except for neutral kaon $K_{L}^{0}$. Then, they scatter off the background nucleons by the threshold cross sections only for the exothermic reactions and extraordinarily, can interconvert $p$ and $n$ strongly with each other through the hadron-nucleon interaction even after the freeze-out time of the neutron to proton ratio $n / p$. At that time it is expected that the background proton tends to be changed into a neutron through the strong interaction since protons are more abundant than neutrons, and $n / p$ tends to increase. As a result, in particular, the abundance of ${ }^{4} \mathrm{He}$ dramatically increases because it is the most sensitive to the freeze-out value of $n / p$. Then, we can constrain the abundance of $X$ and obtain the information of 
$\tau_{X}$ from the observational light element abundances.

Here we have studied the hadron injections by using the JETSET 7.4 Monte Carlo event generator [8] to quantitatively understand the hadron jets to agree with the collider experiments [27]. Thanks to the treatments, we can estimate the number of the emitted hadrons as a function of the energy of jets, i.e., as a function of the mass of $X$, even in the regions where there is no experimental data. In addition we can also obtain the energy spectrum of the emitted $K_{L}^{0}$ for various masses of $X$. This is very important in the computations because $K_{L}^{0}$ is never stopped in the electromagnetic plasma, and we should know the energy distributions of $K_{L}^{0}$ 's. On the other hand, we also have estimated the energy-dependent cross sections for $K_{L}^{0}$-nucleon scattering using the existing experimental data $[27,30]$. With this data, we could properly include the hadron-injection effects in BBN computations.

To estimate the theoretical errors, we performed Monte Carlo simulation including the theoretical uncertainties which come from those of the elementary nuclear reaction rates and hadron-nucleon interaction rates. To obtain the degree of agreements between theory and observation, we performed the maximum likelihood method and the $\chi^{2}$ fitting including both the observational and theoretical errors. To correctly compare each model in the various parameters $(\eta$, $\tau_{X}$, and $\left.n_{X} / s\right)$, the above procedure is quite crucial because a constraint which is obtained when we intentionally fix the parameters has little meaning.
As a result, we have obtained the upper bound on the abundance $n_{X} / s$ as a function of the lifetime $\tau_{X}$ to agree with the observations for the wide range of the mass $m_{X}$ $=10 \mathrm{GeV}-100 \mathrm{TeV}$ which is relevant for various models of supergravity or superstring theory. However, we might have underestimated the deuterium abundances where the lifetime is longer than $\mathcal{O}\left(10^{2}\right)$ sec because deuterium can be produced by the destruction of ${ }^{4} \mathrm{He}$ by the high-energy free neutrons, i.e., "hadrodissociation" effects which we ignored in this work. Therefore, if the process is effective, that would mean we obtained the conservative upper bounds for only $\tau_{X} \geq \mathcal{O}\left(10^{2}\right)$ sec. In a separate paper, we will comprehensively study the subject [28]. We have also applied the results obtained by a generic hadronic decaying particle to gravitino $\psi_{3 / 2}$. Then we have gotten the upper bound on the reheating temperature after primordial inflation as a function of the mass, $T_{R} \lesssim 10^{7}-10^{8} \mathrm{GeV}\left(T_{R} \lesssim 10^{8}-10^{9} \mathrm{GeV}\right)$ for $m_{3 / 2}$ $=10-100 \mathrm{TeV}$ at $95 \%$ C.L. in the case of low D (high D).

\section{ACKNOWLEDGMENTS}

The author wishes to thank T. Asaka, M. Kawasaki, K. Maki, T. Moroi, and J. Yokoyama for valuable discussions and suggestions. He also thanks J. Arafune, O. Biebel, S. Mihara, and M. M. Nojiri for useful comments.
[1] J. Ellis, G.B. Gelmini, J.L. Lopez, D.V. Nanopoulos, and S. Sarkar, Nucl. Phys. B373, 399 (1992).

[2] M. Kawasaki and T. Moroi, Prog. Theor. Phys. 93, 879 (1995).

[3] E. Holtmann, M. Kawasaki, K. Kohri, and T. Moroi, Phys. Rev. D 60, 023506 (1999).

[4] M. Kawasaki, K. Kohri, and T. Moroi, Phys. Rev. D 63, 103502 (2001).

[5] M.H. Reno and D. Seckel, Phys. Rev. D 37, 3441 (1988).

[6] M. Kawasaki, K. Kohri, and N. Sugiyama, Phys. Rev. D 62, 023506 (2000).

[7] K. Kohri and J. Yokoyama, Phys. Rev. D 61, 023501 (1999).

[8] T. Sjöstrand, Comput. Phys. Commun. 82, 74 (1994).

[9] S. Dimopoulos, R. Esmailzadeh, L.J. Hall, and G.D. Starkman, Astrophys. J. 330, 545 (1988); Nucl. Phys. B311, 699 (1989).

[10] J.M. O'Meara, D. Tytler, D. Kirkman, N. Suzuki, J.X. Prochaska, D. Lubin, and A.M. Wolfe, astro-ph/0011179.

[11] S. Burles and D. Tytler, Astrophys. J. 507, 732 (1998).

[12] J.K. Webb et al., Nature (London) 388, 250 (1997).

[13] D. Kirkman, D. Tytler, J.M. O'Meara, S. Burles, D. Lubin, N. Suzuki, R.F. Carswell, M.S. Turner, and E.J. Wampler, astro-ph/0103305.

[14] B.D. Fields and K.A. Olive, Astrophys. J. 506, 177 (1998).

[15] P. Bonifacio and P. Molaro, Mon. Not. R. Astron. Soc. 285, 847 (1997).

[16] B.D. Fields, K. Kainulainen, K.A. Olive, and D. Thomas, New Astron. 1, 77 (1996).

[17] S.G. Ryan, J. Norris, and T.C. Beers, Astrophys. J. 523, 654 (1999).

[18] S.G. Ryan, T.C. Beers, K.A. Olive, B.D. Fields, and J. Norris,
Astrophys. J. Lett. 530, L57 (2000).

[19] NACRE Collaboration, C. Angulo et al., Nucl. Phys. A656, 3 (1999).

[20] R.H. Cyburt, B.D. Fields, and K.A. Olive, New Astron. 6, 215 (1996).

[21] M.S. Smith, L.H. Kawano, and R.A. Malaney, Astrophys. J., Suppl. 85, 219 (1993).

[22] L.H. Kawano, “LET'S GO: EARLY UNIVERSE II, Primordial Nucleosynthesis, The Computer Way," FERMILAB-Pub92/04-A (1992).

[23] C. Brune et al., Phys. Rev. C 60, 015801 (1999).

[24] G.M. Hale et al., ENDF/B-VI Evaluation, Material 125, Revision 1 (1991).

[25] K.M. Nollett and S. Burles, Phys. Rev. D 62, 123505 (2000).

[26] E. Vangioni-Flam, A. Coc, and M. Cassé, Astron. Astrophys. 360, 15 (2000).

[27] Particle Data Group, D.E. Groom et al., Eur. Phys. J. C 15, 1 (2000).

[28] M. Kawasaki, K. Kohri, and T. Moroi (in preparation).

[29] W.E. Cleland et al., Phys. Rev. D 12, 1247 (1975).

[30] Durham Database Group, http://www-spires.dur.ac.uk/hepdata/ reac.html

[31] G.F. Giudice, A. Riotto, and I. Tkachev, J. High Energy Phys. 08, 009 (1999); 11, 036 (1999).

[32] R. Kallosh, L. Kofman, A. Linde, and A.V. Proeyen, Class. Quantum Grav. 17, 4269 (2000).

[33] D.H. Lyth and H.B. Kim, hep-ph/0011262.

[34] H.P. Nilles, M. Peloso, and L. Sorbo, Phys. Rev. Lett. (to be published), hep-ph/0102264. 